\title{
Prevalence of methicillin-resistant Staphylococcus aureus (MRSA) in retail food in Singapore
}

\author{
Kyaw Thu Aung 1,5, Li Yang Hsu², Tse Hsien Koh ${ }^{3}$, Hapuarachchige Chanditha Hapuarachchi ${ }^{1}$, Man Ling Chau', \\ Ramona Alikiiteaga Gutiérrez ${ }^{1 *}$ and Lee Ching $\mathrm{Ng}^{1,4}$
}

\begin{abstract}
We characterised 227 Staphylococcus aureus isolates from retail food and food handlers' gloves samples obtained through food surveillance and risk assessment studies between 2011 and 2014. Of 227 isolates, five (2.2\%) were methicillin-resistant and belonged to sequence types ST80 $(n=3)$ and ST6 $(n=2)$. All five isolates belonged to SCCmec type IV, were Panton-Valentine leukocidin (pvl)-negative and staphylococcal enterotoxin genes-positive. Resistance to azithromycin was found in ST80 isolates, in addition to resistance to beta-lactams. Our finding of two clinically relevant methicillin-resistant S. aureus (MRSA) strains (ST80 and ST6) in ready-to-eat food and food contact surfaces at retail in Singapore suggests food and food contact surfaces as potential environmental sources of MRSA in the community.
\end{abstract}

Keywords: Methicillin-resistant Staphylococcus aureus (MRSA), Retail food, Food contact surface, Antibiotic resistance, Enterotoxin genes, Panton-valentine leukocidin (pvl) gene

\section{Dear Editor,}

Methicillin-resistant Staphylococcus aureus (MRSA) has been recognised as an important nosocomial pathogen and has reportedly been associated with foodborne illnesses [1-3]. MRSA has also recently been listed as one of the high-priority antibiotic-resistant pathogens as ranked by the World Health Organisation. In Singapore, surveillance and control programmes of MRSA have been established in various hospitals $[4,5]$. However, there is a substantial lack of information on the prevalence of MRSA in food, including at retail. Such information would be useful to better understand the risk of exposure to MRSA through food, particularly ready-to-eat food, in contrast with the more typical known transmission route via contact. Our findings provide preliminary insights on the extent of the spread of MRSA in ready-to-eat food in Singapore.

In this study, we characterised 227 coagulase-positive Staphylococcus aureus strains isolated from retail food and swabs taken from food handlers' gloves. These

\footnotetext{
* Correspondence: Ramona_GUTIERREZ@nea.gov.sg

'Environmental Health Institute, National Environment Agency, 11 Biopolis

Way, \#04-03/04, Helios Block, Singapore 138667, Singapore

Full list of author information is available at the end of the article
}

isolates and samples were obtained from food surveillance and risk assessment studies conducted between 2011 and 2014. The isolates were confirmed to be $S$. aureus using coagulase rabbit plasma (Remel), and conventional PCR for the detection of $f e m A$ gene with modified primers (F- GATCATTTATGGAAGATACG TCAG, R- GATAAAGAAGAAACCAGCAGAGATAG) and PCR conditions from previous studies $[6,7]$. To understand the occurrence of methicillin resistance among these $S$. aureus isolates, we used mecA-PCR for the detection of MRSA, followed by PBP2 latex agglutination test (Oxoid) and disc diffusion with Cefoxitin $30 \mu \mathrm{g}$ for confirmation. MRSA strains were further characterised by Multi-Locus Sequence Typing (MLST), staphylococcal Protein A (spa) typing, and staphylococcal cassette chromosome (SCCmec) typing. Strains were also analysed for the presence of virulence genes: staphylococcal enterotoxin (sea, seb, sec, sed, see, seg, seh, sei, sej, sek, sel, sem, seo and sep), Panton-Valentine leukocidin ( $p v l)$, exfoliative toxin (eta, etb and etd), and toxic shock syndrome toxin (tsst-1) genes [7, 8]. Antibiotic susceptibility testing was performed and interpreted according to the Clinical and Laboratory Standards Institute (CLSI) guideline [9]. 


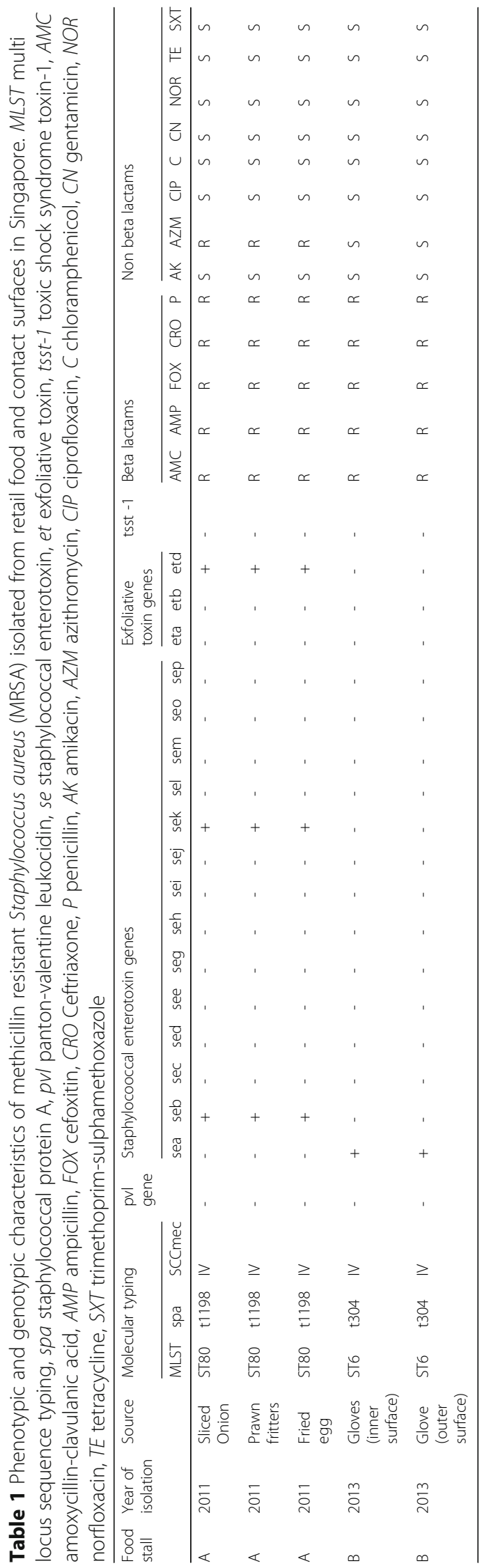


Of the 227 S. aureus isolates, five (2.2\%) from two food stalls were methicillin-resistant (Table 1) and belonged to Sequence Types (ST) 80 (spa type t1198) (isolated from sliced onion, prawn fritters, fried egg) and ST6 (spa type t304) (from swabs of food handlers' gloves) [7]. All five isolates belonged to SCCmec type IV, and were $p v l$-negative. Unlike SCCmec types I-III strains which are often associated with nosocomial infections, strains belonging to SCCmec type IV are capable of colonising healthy individuals and may be community-associated. Some SCCmec type IV strains may be associated with livestock, suggesting a possible transmission between human and food-producing animals [10-12]. In Singapore, community-associated (CA) MRSA strains previously reported were isolated from clinical samples and belonged mainly to ST30, ST59 and ST772 [13]. To our knowledge, no ST6 strain has been reported in Singapore, whereas a ST80 CA-MRSA strain was previously reported in a local hospital in 2003, from a patient with a chin abscess [8]. S. aureus ST80, belonging to Clonal Complex 80 (CC80), was first reported in Denmark in 1993 and has been recognised as a major clone of CA-MRSA, widely spread across Europe [14-16]. Most ST80 strains reported elsewhere belong to SCCmec type IV, are $p v l$-positive, and are usually associated with severe skin/soft tissue infections and necrotising pneumonia [17]. Nonetheless, $p v l$-negative ST80 strains have also been reported in clinical cases overseas $[18,19]$. ST6 MRSA is a doublelocus variant of CC5, which is one of the five CCs from which major epidemic MRSA isolates are believed to have emerged [20]. ST6 MRSA was previously reported in human cases and carriers in Australia, Oman and United Arab Emirates (UAE) [16, 21, 22]. In particular, ST6 SCCmec type IV (t304) pvl-negative MRSA, a strain susceptible to non-beta-lactams, was previously isolated from patients overseas, suggesting that the ST6 strain isolated in our study may be capable of causing human infection [23]. In addition, CC6-ST6-t304 strains have previously been isolated from human (MSSA and MRSA), feral cat (MRSA) and camel (MSSA) suggesting that the strain may be transmissible across different host species [19, 24-26].

In this study, we detected enterotoxin genes $s e b, s e k$, and exfoliative toxin gene etd in ST80 strains; and enterotoxin gene sea in ST6 strains [7]. No other toxin genes were detected. Enterotoxins sea and seb are known to cause approximately $90 \%$ of staphylococcal food poisoning worldwide [27]. Enterotoxin sek is considered a staphylococcal enterotoxin-like protein, though its ability to cause food poisoning has yet to be demonstrated [28]. etd is an exfoliative toxin serotype known to be associated with mild forms of cutaneous infections such as abscesses and furuncles [29]. The presence of enterotoxin genes (sea and seb) in these MRSA isolates suggests the isolates' potential to produce toxins, and cause staphylococcal food poisoning, if allowed to grow in sufficient numbers in food. Antimicrobial susceptibility results showed that all 5 MRSA isolates were phenotypically susceptible to amikacin, ciprofloxacin, chloramphenicol, gentamicin, norfloxacin, tetracycline, and trimethoprim/sulfamethoxazole. Susceptibility to at least 3 non-beta-lactams in MRSA is used as a proxy to define community-associated strains [30]. We found ST80 isolates resistant to azithromycin in addition to the beta-lactams tested (Table 1). An emerging trend of macrolide resistance has been reported in CA-MRSA from the human and livestock sectors [11, 31]. MRSA with resistance to additional antibiotic classes is a concern, and may reflect the increasing use of these antibiotics in the local clinical practice, which warrants further investigation.

In conclusion, we report two clinically relevant MRSA strains (ST80 and ST6) in ready-to-eat food and food contact surfaces at retail. Humans (food handlers), rather than animals, were likely the sources of contamination. Our limited findings suggest ready-to-eat food and food contact surfaces as potential environmental sources for colonisation and spread of MRSA in the community. To date, little is known about the transmission of MRSA infections through food and food contact surfaces, however their possible roles in the dissemination of specific MRSA lineages cannot be ruled out. The data warrant a more comprehensive and integrated (farm-to-hospital approach) surveillance of MRSA in Singapore and elsewhere.

\section{Abbreviations}

AK: Amikacin; AMC: Amoxycillin-clavulanic acid; AMP: Ampicillin; AZM: Azithromycin; C: Chloramphenicol; CA-MRSA: Community-associated methicillin-resistant Staphylococcus aureus; CIP: Ciprofloxacin; CN: Gentamicin; CRO: Ceftriaxone; Et: Exfoliative toxin; FOX: Cefoxitin; mecA: Gene encoding methicillin-resistant-S. aureus-specific-penicillin-binding protein; MLST: MultiLocus Sequence Typing; MRSA: Methicillin-resistant Staphylococcus aureus; NOR: Norfloxacin; P: Penicillin; PBP2: Penicillin binding protein 2; PVI: Pantonvalentine leukocidin; SCCmec: Staphylococcal cassette chromosome; Se: Staphylococcal enterotoxin; Spa: Staphylococcal Protein A; ST: Sequence type; SXT: Trimethoprim-Sulphamethoxazole; TE: Tetracycline; Tsst: Toxic shock syndrome toxin

\section{Acknowledgements}

Not applicable.

\section{Funding}

This study was funded by the National Environment Agency, Singapore.

Availability of data and materials

Please contact corresponding author for data requests.

\section{Authors' contributions}

KTA, LYH, HCH and RAG were involved in the conception and design of the study. KTA, MLC and THK performed the identification and characterisation of isolates. KTA, LYH, THK, HCH, RAG and LCN were involved in the analysis and interpretation of the data. All authors read and approved the final manuscript. 


\section{Ethics approval and consent to participate}

Not applicable.

\section{Consent for publication}

Not applicable.

\section{Competing interests}

The authors declare that they have no competing interests.

\section{Publisher's Note}

Springer Nature remains neutral with regard to jurisdictional claims in published maps and institutional affiliations.

\section{Author details}

'Environmental Health Institute, National Environment Agency, 11 Biopolis Way, \#04-03/04, Helios Block, Singapore 138667, Singapore. ${ }^{2}$ Department of Infectious Diseases, Tan Tock Seng Hospital, 11 Jalan Tan Tock Seng, Singapore 308433, Singapore. ${ }^{3}$ Diagnostic Bacteriology, Pathology Department, Singapore General Hospital, 20 College Road, Academia, Level 7, Diagnostic Tower, Singapore 169856, Singapore. ${ }^{4}$ School of Biological Sciences, College of Science, Nanyang Technological University, 60 Nanyang Drive, Singapore 657551, Singapore. ${ }^{5}$ School of Chemical and Biomedical Engineering, College of Engineering, Nanyang Technological University, 62 Nanyang Drive, Singapore 637459, Singapore.

Received: 7 June 2017 Accepted: 4 September 2017

\section{Published online: 08 September 2017}

\section{References}

1. Alexandra F, Britta K, Gladys K, Beatriz G-R, Katja A, Jens-Andre H, Annemarie K, Juliane B, Bernd A, Bernd-Alois T. Methicillin susceptible and resistant Staphylococcus aureus from farm to fork impact on food safety. Tehnologija mesa. 2011:1:60-5.

2. Jones TF, Kellum ME, Porter SS, Bell M, Schaffner W. An outbreak of communityacquired foodborne illness caused by methicillin-resistant Staphylococcus aureus. Emerg Infect Dis. 2002;8(1):82-4.

3. Kluytmans J, van Leeuwen W, Goessens W, Hollis R, Messer S, Herwaldt L, Bruining $H$, Heck M, Rost J, Van Leeuwen N. Food-initiated outbreak of methicillin-resistant Staphylococcus aureus analysed by pheno- and genotyping. J Clin Microbiol. 1995;33(5):1121-8.

4. Hsu LY, Koh TH, Tan TY, Ito T, Ma XX, Lin RT, et al. Emergence of communityassociated methicillin-resistant Staphylococcus aureus in Singapore: a further six cases. Singap Med J. 2006;47(1):20-6.

5. Tambyah PA, Kumarasinghe G. Methicillin-resistant Staphylococcus aureus control at the National University Hospital, Singapore: a historical perspective. Ann Acad Med Singap. 2008:37(10):855-60.

6. Veras JF, Carmo LSD, Tong LC, Shupp JW, Cummings C, Santos DAD, Cerqueira MMOP, Cantini A, Nicoli JR, Jett M. A study of the enterotoxigenicity of coagulase-negative and coagulase-positive staphylococcal isolates from food poisoning outbreaks in Minas Gerais, Brazil. Int J Infect Dis. 2008:12:410-5.

7. Aung KT, Lo JACY, Chau ML, Kang JSL, Yap HM, Gutiérrez RA, Yuk H-G, Ng LC. Microbiological safety assessment and risk mitigation of Indian rojak (deep fried ready-to-eat food) in Singapore. Southeast Asian J Trop Med Public Health. 2016;47(6):1231-45.

8. Hsu LY, Tristan A, Koh TH, Bes M, Etienne J, Kurup A, Tan TT, Tan BH. Community-associated methicillin-resistant Staphylococcus aureus. Singapore Emerg Infect Dis. 2005;11(2):341-2.

9. Clinical and Laboratory Standards Institute. Performance standards for antimicrobial susceptibility testing: seventeenth informational supplement. Clinical and Laboratory Standards Institute CLSI document. (2007). M100MS17 [ISBN 1-56238-625-5].

10. Nemati M, Hermans K, Lipinska U, Denis O, Deplano A, Struelens M, Devriese LA, Pasmans F, Haesebrouck F. Antimicrobial resistance of old and recent Staphylococcus aureus isolates from poultry: first detection of livestockassociated methicillin resistant strain ST398. Antimicrob Agents Chemother. 2008:52(10):3817-9.

11. Cavaco LM, Miragaia M, Rolo J, Conceicao T, Hasman H, Aarestrup FM, De Lencastre $\mathrm{H}$. Comparison between livestock and community associated MRSA in Europe. Poster session presented at $3^{\text {rd }}$ ASM conference on antimicrobial resistance in Zoonotic bacteria and Foodborne pathogens in animals, humans and the environment, Aix-en-Provence. France.
12. International Working Group on the Classification of Staphylococcal Cassette Chromosome Elements (IWG-SCC). Classification of staphylococcal cassette chromosome mec (SCCmec): guidelines for reporting novel sCCmec elements. Antimicrob Agents Chemother. 2009:53(12):4961-7.

13. Wijaya L, Hsu LY. Community-associated methicillin-resistant Staphylococcus aureus skin and soft tissue infections. Proc Singapore Healthc. 2010;19(3):212-9.

14. Faria NA, Oliveira DC, Westh $H$, Monnet DL, Larsen AR, Skov R, De Lencastre $H$. Epidemiology of emerging methicillin-resistant Staphylococcus aureus (MRSA) in Denmark: a nationalwide study in a country with low prevalence of MRSA infection. J Clin Microbiol. 2005;43:1836-42.

15. Stam-Bolink EM, Mithoe D, Baas WH, Arends JP, Moller AV. Spread of a methicillin-resistant Staphylococcus aureus ST80 strain in the community of the northern-Netherlands. Eur J Clin Microbiol Infect Dis. 2007:26:723-7.

16. Deurenberg RH, Stobberingh EE. The evolution of Staphylococcus aureus. Infect Genet Evol. 2008:8:747-63.

17. Budimir A, Deurenberg RH, Bosnjak Z, Stobberingh EE, Cetkovic H, Kalenic S. A variant of the southern German clone of methicillin-resistant Staphylococcus aureus is predominant in Croatia. Clin Microbiol Infect. 2010;16(8):1077-83.

18. Djoudi F, Bonura C, Benallaoua S, Touati A, Touati D, Aleo A, Cala C, Fasciana T, Mammina C. Panton-valentine leukocidin positive sequence type 80 methicillin-resistant Staphylococcus aureus carrying a staphylococcal cassette chromosome mec type IVc is dominant in neonates and children in an Algiers hospital. New Microbiol. 2013;36:49-56.

19. Monecke S, Coombs G, Shore AC, Coleman DC, Akpaka P, Borg M, Chow H, Ip M, Jatzwauk $L$, et al. A field guide to pandemic, epidemic and sporadic clones of methicillin-resistant Staphylococcus aureus. PLoS One. 2011;6(4):e17936.

20. Enright MC, Robinson DA, Randle G, Feil EJ, Grundmann H, Spratt BG. The evolutionary history of methicillin-resistant Staphylococcus aureus (MRSA). Proc Natll Acad Sci USA. 2002;99(11):7687-92.

21. Monecke S, Skakni L, Hasan R, Ruppelt A, Ghazal SS, Hakawi A, Slickers P, Ehricht R. Characterisation of MRSA strains isolated from patients in a hospital in Riyadh, Kingdom of Saudi Arabia. BMC Microbiol. 2012;12:146-54

22. Weber S, Ehricht R, Slickers P, Abdel-Wareth L, Donnelly G, Pitout M, Stefan M. Genetic fingerprinting of MRSA from Abu Dhabi. Vienna. Eur J Clin Microbiol Infect Dis. 2010;2010

23. Udo EE, Al-Lawati BA-H, Al-Muharmi Z, Thukral SS. Genotyping of methicillin-resistant Staphylococcus aureus in the Sultan Qaboos University Hospital, Oman reveals the dominance of Panton-valentine leucocidinnegative ST6-IV/t304 clone. New Microbes New Infect. 2014;2(4):100-5.

24. Sonnevend Á, Blair I, Alkaabi M, Jumaa P, Al Haj M, Ghazawi A, Akawi N, Jouhar FS, Hamadeh MB, et al. Change in methicillin resistant Staphylococcus aureus clones at a tertiary care hospital in the United Arab Emirates over a 5-year period. J Clin Pathol. 2012;65:178-82.

25. Harastani HH, Tokajian ST. Community-associated methicillin-resistant Staphylococcus aureus clonal complex 80 type IV (CC80-MRSA-IV) isolated from the Middle East: a heterogeneous expanding clonal lineage. PLoS One. 2014;9:1-11

26. Bierowiec K, Ploneczka-Janeczko K, Rypula K. Is the colonisation of Staphylococcus aureus in pets associated with their close contact with owners? PLoS One. 2016;11(5):e0156052.

27. Pinchuk IV, Beswick EJ, Reyes VE. Staphylococcal enterotoxins. Toxins (Basel). 2010;2(8):2177-97.

28. Argudin MA, Mendoza MC, Rodicio MR. Food poisoning and Staphylococcus aureus enterotoxins. Toxins (Basel). 2010;2(7):1751-73.

29. Yamasaki O, Tristan A, Yamaguchi T, Sugai M, Lina G, Bes M, Vandenesch F, Etienne J. Distribution of the exfoliative toxin D gene in clinical Staphylococcus aureus isolates in France. Clin Microbiol Infect. 2006:12(6):585-8.

30. David MZ, Daum RS. Community-associated methicillin-resistant Staphylococcus aureus: epidemiology and clinical consequences of an emerging epidemic. Clin Microbiol Rev. 2010;23(3):616-87.

31. Como-Sabetti K, Harriman KH, Buck JM, Glennen A, Boxud DJ, Lynfield R. Community-associated methicillin-resistant Staphylococcus aureus: trends in case and isolate characteristics from six years of prospective surveillance. Public Health Rep. 2009:124(3):427-35. 